\title{
An Efficient Existentially Unforgeable Signature Scheme and its Applications
}

\author{
Cynthia Dwork ${ }^{1}$ and Moni Naor ${ }^{2}$ * \\ 1 IBM Almaden Research Center, USA. e-mail:dwork@almaden.ibm.com \\ 2 Weizmann Institute, Israel. e-mail: naor@wisdom.weizmann.ac.il
}

\begin{abstract}
We present a practical existentially unforgeable signature scheme and point out applications where its application is desirable.

A signature scheme is existentially unforgeable if, given any polynomial (in the security parameter) number of pairs

$$
\left(m_{1}, S\left(m_{1}\right)\right),\left(m_{2}, S\left(m_{2}\right)\right), \ldots\left(m_{k}, S\left(m_{k}\right)\right)
$$

where $S(m)$ denotes the signature on the message $m$, it is computationally infeasible to generate a pair $\left(m_{k+1}, S\left(m_{k+1}\right)\right)$ for any message $m_{k+1} \notin\left\{m_{1}, \ldots m_{k}\right\}$. We have developed a signature scheme that requires at most 6 times the amount of time needed to generate a signature using RSA (which is not existentially unforgeable).
\end{abstract}

\section{Introduction}

Consider the problem of providing a "receipt" for data stored in a document repository, where the data can be of arbitrary form, much as one is provided with a claim check at a left luggage counter. In the most simple implementation, the receipt should just be a pair consisting of an identifier and a signature on this identifier. If the signature scheme is existentially forgeable, then anyone can produce what appears to be a valid receipt, or claim check; an existentially unforgeable signature scheme prevents this.

In order to ensure that the retrieved document is authentic, it should be signed by the owner. Suppose, for example, that the document is a will. If the signature is computed using an existentially forgeable scheme, then the will can be challenged by anyone producing a possibly nonsensical "will" and a signature on this "will." However, with an existentially unforgeable signature scheme any signed document, nonsensical or otherwise, has necessarily been signed by the claimed signer.

Our interest in finding efficient existentially unforgeable signature schemes comes from the problem of signing FAXed documents. Since FAXed documents have received legal standing in court, it is essential to find a signature scheme

\footnotetext{
${ }^{*}$ Research Performed when this author was with the IBM Almaden Research Center.
} 
appropriate to this environment. Let $D$ be a document, let $h$ be a collisionintractable hash function, and let $S$ be a signing function. Assume the sender sends to the receiver $D, h(D), S(h(D))$. Even if the receiver can check that the document has been correctly hashed and that the signature on the hash is valid, once $D$ is printed out on a FAX machine there is no way to re-capture $D$ from the printed image. For example, scanning the printed image optically will probably produce some $D^{\prime} \neq D$, since the scanned image may be slightly tilted, or dirty, etc. Either the receiver must have a small disk drive, or even a tape drive on which to store $D$, or some other party must store the data and issue a receipt to the receiver. In particular, the (not necessarily trustworthy) sender could store the data; the receipt could be the pair $(h(D), S(h(D)))$. Since $h(D)$ looks "random," the signature scheme must be existentially unforgeable, otherwise, as in the claim check example, anyone could generate what appears to be a valid receipt ${ }^{3}$.

In this paper we present an efficient existentially unforgeable signature scheme which we believe is the first practical one. The security of the scheme relies only on the RSA assumption: it is computationally infeasible to extract pth roots mod $N$, where $p$ is a prime and $N$ is a product of two large primes. The cost of implementing our scheme is close to that of RSA: for all reasonable parameters, the cost of signing and verifying is only six times that of RSA. Thus in almost every scenario where it is feasible to apply RSA it should be feasible to use our scheme.

The paper is organized as follows: in the next section we describe the history of digital signatures, emphasizing work relevant to our scheme. In Section 3 the scheme is presented. Section 4 provides the outline of the proof of security. In Section 5 we describe how to use the proposed scheme in the context of signing FAXes.

\section{Related Work}

Since the introduction of the concept of digital signatures by Diffie and Hellman $[3]$ and the first proposals of candidates for implementation $[13,17]$ the subject has been widely studied. We briefly outline the major developments (not necessarily in chronological order), especially those pertaining to the scheme proposed in this paper.

Goldwasser, Micali, and Rivest [10] formalized the notion of security of a signature scheme. The highest form of security they proposed was called existentially unforgeable under an adaptive chosen plaintext attack. In this attack the adversary (or forger) gets to see a signature on any message of its choice, in an adaptive manner. The forger has then to produce, without the cooperation of the signature algorithm, a signature on one message that was not previously signed. (See exact definition in [10]; we use their definitions in this paper.) This

\footnotetext{
${ }^{3}$ Actually, at the cost of complicating the protocol, we can assume $h$ is chosen, by the receiver, from a family of universal one-way hash functions [15]. This is discussed further in Section 5.
} 
notion was considered too strong for practical purposes, but as the application to signing FAXed documents briefly sketched in the Introduction shows, it does appear in the "real-world". The RSA [17] and Rabin's [16] schemes are known to be not existentially unforgeable.

The implementation of an existentially unforgeable signature scheme suggested in [10] was based on the hardness of factoring. Constructions based on weaker assumptions (trapdoor permutation, 1-1 one-way functions and one-way functions) were given in $[1,15,18]$. These schemes are all rather inefficient. For instance, the scheme in [10] employs a tree whose height is proportional to the length of the message (or some digest of it). [15, 18], who use Merkle's authentication tree method [12], need a tree whose height is a least the logarithm of the total number of messages signed by the system. Signing and verifying both involve tracing a path from the root to a leaf, where moving from node to node is quite expensive (e.g. an RSA computation in [10], as optimized in [9]).

Our scheme employs a tree as well. However it is very shallow one; in a lifetime of a system it is very unlikely to need more than three levels.

There are several constructions of one-time or fixed-time signature schemes that are existentially unforgeable. (One-time means that the public key is good for one signature only; fixed-time means that there is an a priori upper bound on the number of messages the scheme can sign. The size of the public key is usually related to this number.) These are the original Lamport scheme (described in [3] and used in $[12,15,18]$ ). This requires as many invocations of a one-way function as there are bits to be signed (Some improvements are known). The scheme of Bos and Chaum [2] can be considered as a fixed-time signature scheme. The size of the public information needed grows at least as fast as the square root of the number of messages the scheme should be able to sign. See more about it below.

Even, Goldreich, and Micali [5] tried to combat the computational cost of signature schemes by distinguishing between on-line and off-line computation. Their scheme requires extensive pre-computation, "between" signing of different documents, but the on-line computation required for signing is very efficient. The size of a signature is rather large.

The El-Gamal scheme [4] relies on no cleanly specified function; moreover, given a legitimately signed document in that scheme, it is possible to generate other legitimate signatures and messages and thus is not existentially unforgeable. The scheme of Fiat and Shamir [7], and its descendants [8, 14, 19], are very efficient, since, unlike RSA and related schemes, they do not require modular exponentiation. However, they do require that the "one-way hash" function actually be something stronger, more like a black-box random function (no precise definition of the assumptions needed appeared). None of them is known to be existentially unforgeable.

Most of the ingredients of our scheme have appeared before; the contribution of this work is merely in finding the right mixture that makes the full scheme efficient. The idea of using exponentiation to hide information appears in the original RSA signature scheme [17]. Fiat and Shamir employ the subset product technique for signing [7]. Merkle [12] suggested the tree authentication scheme, 
but in his scheme the tree cannot be shallow. The scheme in [2] is similar in spirit to the one-time version of our scheme used in every node. Bellare and Micali [1] suggested a tree based scheme where nodes are "revived" by choosing a new trapdoor permeation which, in turn, is authenticated by the parent of the current node. Our scheme can be seen as an efficient way of performing this, by replacing the trapdoors of [1] with "masks" from the Fiat-Shamir scheme [7].

\section{The Scheme}

\subsection{Outline}

In rough outline, the scheme works as follows (details are given in the next section). Every signer $s$ has, as in any signature scheme, a pair of keys. The public key is used to verify the signature on messages supposedly signed by $i$. The private key contains information known only to $s$, and is used by $s$ to compute signatures of given messages.

The signer maintains a short, very bushy tree. Every message is associated with a leaf of the tree. Thus, the tree will be quite large; however, as we shall see, at any time the signer need only store information associated with a single root-leaf path in the tree. For concreteness, we take the outdegree of the tree to be $l=1000$. If the tree is of height 3 then it has a billion leaves, (which should be sufficient to sign all the message's $s$ should desire). Associated with every node is a 1000-bit number. This number is random for all internal nodes and a message for the the leaves. Assume all messages have length at most one thousand bits (larger messages may be hashed down to this length or broken into pieces, with each piece signed individually). A signer's public key is an integer $N$, the product of two large primes, and $y_{\text {root }}$, the random number associated with the root of the tree. ( $y_{\text {root }}$ can be the same for all signers, as long as it chosen as a random l-bits number initially.) At first all leaves are unused. To sign a message $m$, the signer associates $m$ with the leftmost unused leaf, say $w$, and authenticates the path from the root to $w$. That is, starting with the root, for each step along the (length 3) path from root to leaf, the signer authenticates the string associated with the child, using the string associated with the parent. This is done via a basic authentication step which involves using subset product [7] and extracting $p$ th roots [17].

Intuitively, the reason the scheme is practical is that we have found a way to "re-use" an internal node many times (once for each leaf in the subtree rooted at this node).

Common to all users (signers and verifiers) are two lists: $P$ contains one thousand primes, and $X$ contains one thousand random strings. Let $v$ be an internal node in the tree, with associated string $y_{v}$, and let $w$ be the $j$ th child of $v$. In the basic authentication step we use the bits of the string associated with $w$ to select elements of $X$. We compute the product of $y_{v}$ and the selected elements. Finally, we compute the $p_{j}$ th root of this product. Note that since the values associated with the internal nodes are all random, there is virtually no 
chance that any two such strings are identical. Thus, we never use the same $y_{v}, p_{j}$ pair twice in a basic authentication step. This is critical in obtaining existential unforgeability.

\subsection{Detailed Description}

Let $l$ be the security parameter of the scheme ( $l$ should be such that it is infeasible to factor $l$-bit numbers). In practice we would take $l$ to be about 1000 , though of course we can take it to be larger. There are two sets $X=\left\{x_{1}, x_{2}, \ldots, x_{l}\right\}$ and $P=\left\{p_{1}, p_{2}, \ldots, p_{l}\right\}$ of integers. The $x_{i}$ 's are random integer of length at most $l$. The $p_{i}$ 's are primes, they can be either the $l$ smallest primes or $l$ random primes of length $l$ (This depends on the RSA assumption we are making, whether it is "it is hard to extract $p$ th roots $\bmod N$ for small $p$ " or "it is hard to extract $p$ th roots $\bmod N$ for random $p "$ ). These lists are fixed and the same for all signers. All signers and verifiers should have access to them.

Each signer maintains a tree of height $d$ and outdegree $l$. As mentioned above, $d$ need not be larger than 3 , since $l^{d}$ is a lower bound on the number of messages it is possible to sign. Associated with each internal node $v$ is an $l$-bit number $y_{v}$. Associated with each leaf is an $l$-bit string representing a message of up to $l$ bits. The value associated with the root, $y_{\text {root }}$, is random and is part of the public information, like $P$ and $X$. The value associated with a non-root internal node is chosen at random and on the fly, and is authenticated in a basic authentication step that uses the (authenticated) value associated with the parent node. The $y$ 's within any given tree must be distinct; there is virtually no chance that the same $y$ will be chosen twice. Similarly, a message, which is conceptually just a value associated with a leaf, is authenticated using the the value stored at the parent node. The full signature of a message is the path from the root to the leaf, together with the basic authentication steps for all values associated with the nodes along the path.

The public key: the public key of each signer $s$ is a number $N_{s}$, where $N_{s}$ is an $l$ bit number which is a product of two primes. It is important to chose the primes at random (and of course independent of the list $X$ ).

The basic authentication step: For the basic authentication step at a nonroot node $v$, let $y_{v}$ be the value associated with $v$ (a message, if $v$ is a leaf), and let $z_{v}$ be the value associated with the parent of $v$. We use the bits of $y_{v}$ as selectors of the elements of $X$. Let $y_{v i}$ denote the $i$ th bit of $y_{v}$, for $1 \leq i \leq l$. Let $v$ be the $j$ th child of its parent. Then $\operatorname{auth}\left(y_{v}\right)$ is given by

$$
\left(z_{v} \prod_{y_{v i}=1} x_{i}\right)^{1 / p_{j}} \bmod N_{s}
$$

The authentication of $y_{v}$ can be verified as follows. Given a string $\alpha$ purported to be auth $\left(y_{v}\right)$, and given also $z_{v}, y_{v}, j$, and the public lists $P$ and $X$, one computes

$$
z_{v} \prod_{y_{v i}=1} x_{i} \bmod N_{s}
$$


and checks that it is equal to $\alpha^{p_{j}} \bmod N_{s}$.

A signature of a message $m$ associated with leaf $w$ is composed of $m$, the path from the root to $w$ (i.e. the indices of the children), and for each node $v$ along the path (excluding the root and including $w$ ), $y_{v}$ (the value associated with $v$ ) and its authentication, as computed in the basic authentication step just described. The signature is verified by verifying the basic authentication steps for all the nodes on the path.

\subsection{Computational Requirements:}

\section{The complexity time of signing}

Signing a message involves

- $d$ RSA computations (i.e. exponentiations $\bmod N_{s}$ )

$-d$ subset multiplications, i.e. multiplying a random subset of $l$ numbers. This is roughly equivalent to modular exponentiation.

Since we can assume that $d=3$, we say that the complexity of the scheme is six times that of RSA. The complexity of verifying a signature is similar.

\section{Size of signature}

A valid signature consists of $\mathrm{d}$ numbers, each $l$ bits long, plus $d$ numbers to describe the path from the root to the leaf which are $\log l$ bits each. Therefore the size of a signature is roughly 6 times that of RSA.

\section{Size of public key and storage requirements}

The size of public key is an l-bits number (similar to RSA). A cost that RSA does not have is the storage of the lists $X$ and $P$, common to all users, which require $2 l^{2}$ bits altogether. This is roughly $1,000,000$ bits which should be accessible to every user. This is feasible if both signer and receiver are a "full" computer, but may be an obstacle in using the scheme in a smartcard environment. However, as smartcards are getting more powerful, storing $128 \mathrm{~K}$ bytes in ROM on a smartcard is not impossible.

Apart from the lists $X$ and $P$, the memory needed to run the signature scheme is not large, one should essentially maintain a path from the root to a leaf, i.e $d \log l$ bits.

\subsection{Remarks on Implementation}

Consider a particular path in the tree. Since the tree is of height 3 , the path has 3 internal nodes: the root, and two others, say, $v$ and $w$. Let $y_{\text {root }}, y_{v}$, and $y_{w}$ be the values associated with these internal nodes. Then, although the first time a message associated with a child of $w$ is signed, the signer must perform the computation needed to authenticate $y_{w}$ using $y_{v}$, this information can be saved and used for the remaining $l-1$ (about 1000) messages associated with children of $w$. Similarly, the authentication of $y_{w}$ using $y_{\text {root }}$ can be re-used $l^{2}$ times (about 1,000,000 times). 
The $p_{i}$ 's may be chosen to be small in order to speed up verification of a signature. Furthermore, Fiat [6] suggested a way of amortizing RSA computation. His method fits very well with our scheme, since we use different roots anyway.

Other suggestions to speed up RSA and the Fiat-Shamir signature scheme are applicable to our scheme as well. The signer can do its computation modulo each of the factors of $N$ separately and combine them using Chinese remaindering. To speed up the subset product one can pre-process the list $X$ and partition $X$ to small sets and for every set compute all products of its subsets. This can decrease significantly the time spent on the subset multiplication.

\section{Security of the Scheme}

Our definition of security is that of Goldwasser, Micali and Rivest [10]. We only sketch why the proposed scheme obeys this definition. The proof of security is by showing that the ability to generate a single $(m, S(m))$ pair, even for a nonsensical $m$, for an $m$ on which the signer was not explicitly asked to sign, violates the RSA assumption: it is computationally infeasible to extract $p$ th roots $\bmod N$, where $p$ is a (random) prime of length $l$ and $N$ is a product of two large primes chosen at random, also of length $l$.

Intuitively, the security of the scheme rests on the important observations made in [20] and [7]:

- Having a black box that computes $x^{1 / p_{1}} \bmod N$ for random $x$ does not help in evaluating $x^{1 / p_{2}} \bmod N$, if $p_{1}$ and $p_{2}$ are relatively prime.

- For numbers $x_{1}, x_{2}, \ldots x_{l}$, for arbitrary subset $S \subset\{1, \ldots, l\}$ and random $y \in Z_{N}$ the value of $\left(y \prod_{i \in S} x_{i}\right)^{1 / p} \bmod N$ yields no information about any of the $x_{i}^{1 / p}$.

Suppose that the scheme can be broken, i.e. there is a algorithm $\mathcal{A}$ that operates in time $T$ and has probability $\rho$ of breaking the scheme. We show that there is an algorithm $\mathcal{B}$ that works in expected time $O(T)$ and can extract pth roots with probability at least $\rho / l^{2}$.

The input to $\mathcal{B}$ is $x, N$ and $p$. The algorithm consists of two phases, a preprocessing phase where the public key and public information are generated, and a simulation phase where the algorithm $\mathcal{A}$ is simulated on the public key generated in the previous phase.

If a tree based scheme (i.e a system where parents vouch for the authenticity of there children) is broken, then there must be the first time an illegitimate value (i.e. a value not authenticated by the signer) is authenticated at some node $w$. we can guess with probability $1 / l$ at which child $1 \leq j \leq l$ of $w$ this will occur. Furthermore, we can guess with probability at least $1 / l$ an index $1 \leq i \leq l$ where the legitimate value authenticated with $w$ and $j$ and the forged value differ.

Preprocessing phase

1. Choose random $1 \leq i \leq l$ and $1 \leq j \leq l$.

2. Set $N_{s}=N$. 
3. Choose random $l$-bit primes $p_{1}, p_{2}, \ldots p_{j-1}, p_{j+1}, \ldots p_{l}$. Set $p_{j}=p$ and let $P=\left\{p_{1}, p_{2}, \ldots, p_{1}\right\}$.

4. To choose $x_{1}, x_{2}, \ldots x_{i-1}, x_{i+1}, \ldots x_{l}$, generate $l-1$ random values in $Z_{N_{t}}$ and raise them to the power $p_{1} \cdot p_{2} \cdots p_{l}$ modulo $N_{s}$. Thus all the $p_{k}$ th roots of the elements $X \backslash\left\{x_{i}\right\}$ are known and can be computed efficiently. Choose $x_{i}$ to be $(r \cdot x)^{p_{1} \cdots p_{j-1} \cdot p_{j+1} \cdots p_{l}} \bmod N_{s}$, for a random $r \in Z_{N_{s}}$. The important thing to note is that given $x_{i}^{1 / p_{j}}$ it is possible to extract $(r \cdot x)^{1 / p_{j}}$ from it (see $[20]$ ) and hence $x$.

5. We now determine $y_{v}$ for all internal nodes $v$ that are ancestors of the first $T$ (the upper bound on the number of steps of $\mathcal{A}$ ) leaves. The tree is constructed bottom up. Our intuition is: given that $v$ is the $j$ th child of its parent, then

- if $y_{v i}=1$ then $z_{v}$ (the value associated with the parent of $v$ ) should be chosen as $\beta_{v}^{p_{1} \cdot p_{2} \cdots p_{i}} / x_{i}$ for random $\beta_{v} \in Z_{N_{s}}$.

- if $y_{v i}=0$, then $z_{v}$ should be chosen as $\beta_{v}^{p_{1}} p_{2} \cdots p_{1}$ for random $\beta_{v} \in Z_{N_{s}}$. Though the above procedure suggests a bottom-up method for choosing the $y_{v}$, we prefer the following two steps description: start with assigning random bits $b_{u} \in\{0,1\}$ as the values of the $y_{u i}$ 's (i.e. the $i$ th bit of $y_{u}$ ) to all the nodes $u$ of the tree. Note that for a leaf $u, b_{u}$ is necessarily a guess, and is correct with probability $1 / 2$. However, all the other $b_{u}$ 's will indeed be the values of $y_{u i}$.

Given an assignment $b_{u}$ to $y_{u i}$ for all node $u$, it is possible to quickly find a solution to the $y_{v}$ 's together with the appropriate $\beta_{v}$ 's satisfying the requirement that $y_{v i}=b_{v}$ 's for all internal nodes $v$ : choose $\beta_{v} \in Z_{N_{s}}$ at random and set $y_{v}$ by the rule above. With probability $1 / 2 y_{v i}=b_{v}$ (i.e. it has the right $y_{v i}$ ). If they are equal, then accept $y_{v}$ and continue with the preprocessing; otherwise, repeat until successful.

6. Fix $y_{\text {root }}$ as the value the above procedure gave to the root.

Before we proceed in describing the simulation we should note that in the description aboye there is an inaccuracy: we choose values $z \in Z_{N}$ at random (where $z$ stands for either the $x_{k}$ 's or the $y_{v}$ 's), whereas in a regular execution of $\mathcal{A}$ it should be that $z$ is a random $l$-bit number. This can be corrected by replacing $z$ by a random $z^{\prime}$ of length $l$ such that $z=z^{\prime} \bmod N$. However, this gives a certain advantage to the $z$ 's such that $z>\left(2^{l} \bmod N\right)($ since there are fewer $z^{\prime}$ 's that $z=Z^{\prime} \bmod N$ ). Hence we reject such $z$ 's (and repeat the process we used to choose it) with probability proportional to their bias - $\frac{2^{l} / N \mid}{2^{2} / N \mid}$. This cannot increase the expected work by more the a factor of 2 .

The simulation

1. present $\mathcal{A}$ with $\left(X, P, N_{s}, y_{\text {root }}\right)$ as determined in the preprocessing phase.

2. Start the simulation of $\mathcal{A}$; at every step $t, \mathcal{A}$ provides the signer with a message $m_{t}$ and requests a signature. The algorithm $\mathcal{A}$ receives as requested a signature on $m_{t}$ using the path to the th leftmost leaf of the tree chosen in the preprocessing. The signature is generated according to the following:

- To handle a node which is not the $j$ th child of its parent is not a problem, since we can extract all $p_{k}$ th roots when $k \neq j$. 
- To handle a node which is a $j$ th child but is an internal node can also be done easily because of the way $y_{v}$ was chosen in step 5 in the preprocessing phase.

- When handling a leaf $u$ that is the $j$ th child of its parent $v$, then with probability $1 / 2$ the incorrect $b_{u}$ was chosen (i.e. not fitting the $i$ th bit of the message $m_{t}$ for which $\mathcal{A}$ requests a signature). In this case

- Rewind $\mathcal{A}$ for $j$ steps back, to a stage just before parent $v$ is used for the first time.

- Choose a new value to $b_{u}$ at random.

- Choose a new value $y_{v}$, where $v$ is the parent of $u$; the value of $y_{v}$ should obey the restriction $b_{v}$ on $y_{v i}$ imposed in the preprocessing step, thus preventing further propagation of the rollback.

As discussed below, the rewinding may increases the expected run-time by a factor of at most two.

Claim 4.1 The distribution the $\mathcal{A}$ in the simulation witnesses (i.e. the signatures) is the same distribution $\mathcal{A}$ sees in a regular execution.

Proof. The lists $X$ is of $l$ numbers uniformly chosen from the $l$-bits numbers. The list $P$ is a list of $l$ primes of length $l$, given that the input $p$ was chosen at random (which is our assumption). All the $y_{v}$ are uniformly distributed l-bits numbers. Hence, by induction on the number of steps the distribution $\mathcal{A}$ witnesses in the simulation is the same as in a regular execution.

Claim 4.2 The expected time to run the simulation is $O(T)$.

Proof. There are two possibilities that may force us to spend more time than $T$ : one is Step 5 of the preprocessing phase where we may fail in choosing $y_{v}$ that satisfies the requirement on $y_{v i}$. However this happens with probability $1 / 2$ and hence doubles the expected amount of work. The second possibility is in Step 2 of the simulation, where we may have to rewind $\mathcal{A}$ for $j$ steps. However, as before, this happens with probability $1 / 2$ and forces an expected increase of $2 j / l \leq 2$ of the work.

Claim 4.3 The probability of success is at least $\rho / l^{2}$.

Proof. Since the $\mathcal{A}$ in our simulation sees the same distribution as in a regular execution, the probability it breaks the system is $\rho$. furthermore, the distribution $\mathcal{A}$ in the simulation sees is independent of $i$ and $j$ chosen by $\mathcal{B}$. If a $\mathcal{A}$ breaks the signatures scheme there is the first time an illegitimate value is authenticated by some node $w$. By illegitimate we mean that the signer in the simulation authenticated a different value at $w$. Hence, with probability at least $1 / l^{2}$ we have that the forgery occurred at a $j$ th child and the value authenticated by the signer and the value forged differ at the $i$ th bit. If this is the case, then we can extract $x^{1 / p} \bmod N$ from the two authenticated values.

We therefore can conclude

Theorem 1. Any algorithm for breaking the provided signature scheme can be used at a similar cost and probability of success to extract modular roots. 


\section{Application to Signing Faxed Documents}

We assume that at least one of the sender or the receiver is using a computerFAX, or CFAX, system. There are three scenarios to consider: CFAX to CFAX (straightforward) CFAX to FAX (interesting), and FAX to CFAX (same as CFAX to CFAX). As mentioned in the Introduction, the CFAX to FAX scenario is interesting because the receiver cannot store the bit-stream image of the FAXed document, and yet this is the data that has been signed. Thus, the sender must store the data, and issue to the receiver some type of receipt.

We next discuss the different scenarios in more detail. We do not assume the existence of a collision-intractable hash function. Rather, we rely only on the existence of a family of universal one-way hash functions [15]: These functions have the following property: Fix a document $D$. Let $h$ be chosen at random from a family of universal one-way hash functions. Then it is computationally infeasible to find a document $D^{\prime}$ such that $h(D)=h\left(D^{\prime}\right)^{4}$.

Impagliazzo and Naor have described a one-way hash function based on subset sum [11]; in particular, breaking the assumed universal one-way hash property of this family of functions is as hard as solving subset sum. They also suggested less efficient schemes based on factoring.

Throughout this section, we assume the existence of an existentially unforgeable signature scheme secure against chosen plaintext attack, such as the one described in Section 3.

\subsection{When the Receiver is a CFAX}

For documents prepared on-line this is straightforward. The receiver doesn't really care who is actually sending the transmission, since when it verifies the signature it knows that the claimed sender actually signed the document originally. The procedure for sending a document $D$ is as follows. The sender chooses at random a universal one-way hash function $h$ hashing documents down to fingerprints of, say, 1000 bits. Letting $K_{S}$ (respectively, $L_{S}$ ) be the public (resp. private) signature key of the sender, the sender sends to the verifier $S$ (the sender's name), $D$ (the document), and $L_{S}(h(D)$ ) (a signed fingerprint of $D$ ).

The properties of $h$ make it virtually impossible for the receiver to find a document $D^{\prime}$ with the same fingerprint as $D$, i.e., satisfying $h\left(D^{\prime}\right)=h(D)^{5}$. Since the signature scheme is existentially unforgeable, it is virtually impossible to produce $L_{S}\left(m^{\prime}\right)$ for any $m^{\prime}$, despite seeing any (polynomial) number of other signed messages (or hashes of messages) $L_{S}\left(m_{1}\right), L_{S}\left(m_{2}\right), \ldots$ without knowing the private key.

\footnotetext{
${ }^{4}$ This is weaker than collision-intractability, which allows $D$ to be chosen after $h$ is known.

${ }^{5}$ Note that, if the sender did not choose $h$ at random, then the properties of UOWHFs do not prevent finding such a $D^{\prime}$. However, the sender is protected by choosing $h$ at random, and the receiver is protected because it is the responsibility of the receiver to store and later to produce $D$ together with $h(D)$.
} 
If the document is sent as a series of blocks, or pages, then each page must have an identifying header and a page number, as in all legal documents (e.g., "Page 2 of 5"). The identifying nonce can include things like time of day.

Physical documents can be scanned into the sender's machine and treated from then on as if prepared on-line.

\subsection{The Procedure for CFAX to FAX}

Here we have a CFAX machine $C$ sending a document to an ordinary FAX $F$. In this scenario the receiver cannot alone convince a third party that the document was signed by the claimed sender, but it can show to a third party a signed statement promising cooperation in the judgment process. This weakness can be circumvented by adding a tape drive to the box. The idea is that the tapes are used only if the sender refuses to cooperate. As pointed out below, refusal on the part of the sender to cooperate may actually permit the receiver to forge, so it is definitely in the sender's interest to cooperate. In the following, we do not assume that the receiver has a long-term storage device.

To avoid having to maintain and access a directory of public keys, there can be a central agency with whom public keys are registered. The central agency has its own pair of keys, $K_{C e n t e r}, L_{C e n t e r}$, where all users know $K_{C \text { enter }}$ (rather than having to know all public keys).

The CFAX sender, $C$, first forwards to the recipient the statement $L_{C e n t e r}$ (" $C$ 's public signature key is $K_{C}$ "), where $L_{C e n t e r}$ is the private key of the trusted center. The recipient knows $K_{C e n t e r}$ and can therefore be certain of using the correct public key for $F$. The agents proceed as follows.

1. $F$ chooses at random a UOWHF $h$. It does not reveal this to $C$.

2. $C$ sends to $F$ the document $D ; F$ hashes $D$ on-line, computing $h(D)$ and temporarily saving this; it also prints $D$.

3. $F$ sends $h$ to $C$.

4. $C$ computes $h(D)$ and sends $L_{C}(h), L_{C}(h(D))$ to $F$.

5 . Let the pair received by $F$ be $\alpha, \beta$. $F$ checks that $h$ is indeed the function it sent to $C$ above. It verifies that $\alpha=L_{C}(h)$. It verifies that $\beta=$ $L_{C}(h(D))$, using $h(D)$ that it computed and stored above. $F$ then prints $h, L_{C}(h), h(D), L_{C}(h(D))$ in hex or a bar code.

For particularly important transactions $C$ may store $D$ on tape. This is discussed next.

Handling Disputes The tape should be used only as a last resort. Since the signature scheme is existentially unforgeable, the tuple $h, L_{C}(h), h(D), L_{C}(h(D))$ constitutes a promise by $C$ to produce a document that hashes to $h(D)$, since $F$ cannot generate the first two components of the triple because $h$ is chosen at random. Moreover, $D$ was fixed by $C$ without knowledge of $h$, so the only satisfactory document that $C$ can produce is $D$. We are relying here on the 
properties of universal one-way hash functions: If $D$ is fixed and $h$ is chosen at random, then it is "impossible" to find a $D^{\prime}$ such that $h\left(D^{\prime}\right)=h(D)$.

If the sender $C$ refuses to produce $D$, then if $D$ has been stored on tape $F$ can produce the tape. However, since $F$ is the one that chose $h$ this exposes $C$ to "forgery," in that if $F$ had been dishonest and chosen $h$ dependent on $D$ (after Step 2, rather than before Step 2), then $F$ may be able to produce $D^{\prime} \neq D$ such that $h\left(D^{\prime}\right)=h(D)$.

\section{References}

1. M. Bellare and S. Micali, How to Sign Given Any Trapdoor Function, Proc. 20th ACM Annual Symposium on the Theory of Computing, 1988, pp.32-42.

2. J. Bos and D. Chaum, Provably Unforgeable Signatures, Proc. Advances in Cryptology - Crypto'92 Proceedings, Springer Verlag, 1993, pp. 1-14.

3. W. Diffie and M. Hellman, New Directions in Cryptography, IEEE Trans. on Information Theory 22(6), 1976, pp. 644-654.

4. T. El Gamal, A Public Key Cryptosystem and a Signature Scheme Based on Discrete Logarithms, IEEE Trans. Inform. Theory, IT-31(4), 1985, pp. 469-472

5. S. Even, O. Goldreich, and S. Micali, On-line/Off-line Digital Signatures, Proc. Advances in Cryptology - Crypto '89, Springer Verlag, pp. 263-275, 1990.

6. A. Fiat, Batch RSA, Proc. Advances in Cryptology - Crypto '89, Springer Verlag, 1990.

7. A. Fiat and A. Shamir, How to Prove Yourself, Proc. of Advances in Cryptology Crypto '86, Springer Verlag, 1987, pp. 641-654.

8. A. Fiat and A. Shamir, Method, Apparatus, and Article for Identification and Signature, United States Patent 4,748,668 (5/31/88)

9. O. Goldreich, Two Remarks Concerning the Goldwasser-Micali-Rivest Signature Scheme, Proc. Advances in Cryptology - Crypto '86, Springer Verlag, 1987.

10. S. Goldwasser, S. Micali, and R. Rivest, A Digital Signature Scheme Secure Against Adaptive Chosen-Message Attacks, SIAM J. Computing 17(2), pp. 281-301, 1988.

11. R. Impagliazzo and M. Naor, Efficient Cryptographic Schemes Provably as Secure as Subset Sum, Proc. of the 30th Symp. on Foundations of Computer Science, 1989, pp. 236-241. Full version: Technical Report CS93-12, Weizmann Institute.

12. R. Merkle, A Digital Signature Based on a Conventional Encryption Function, Proc. Advances in Cryptology - Crypto '87, Springer Verlag, 1988, pp. 369-378.

13. R. C. Merkle and M. Hellman, Hiding information and Signature in Trapdoor Knapsack, IEEE Transaction on Information Theory, Vol 24, 1978, pp. 525-530.

14. S. Micali and A. Shamir, An Improvement of the Fiat-Shamir Identification and Signature Scheme, Proc. Advances in Cryptology - Crypto '88, LNCS 403, SpringerVerlag, pp. 244-247, 1990

15. M. Naor and M. Yung, Universal One Way Hash Functions and Their Cryptographic Applications, Proc. 21st ACM Annual Symposium on the Theory of Computing, 1989, pp. 33-43.

16. M. O. Rabin Digital Signatures and Public Key Functions as Intractable as Factoring, Technical Memo TM-212, Lab. for Computer Science, MIT, 1979.

17. R. Rivest, A. Shamir, and L. Adelman, A Method for Obtaining Digital Signature and Public Key Cryptosystems, Comm. of ACM, 21 (1978), pp. 120-126. 
18. J. Rompel, One-way Function are Necessary and Sufficient for Signatures, Proc. 22nd ACM Annual Symposium on the Theory of Computing, 1990, pp. 387-394.

19. C. P. Schnorr, Efficient Signature Generation by Smart Cards, J. Cryptology 4, pp. 161-174, 1991.

20. A. Shamir, On the Generation of Cryptographically Strong Pseudo-Random Number Sequences, ACM Trans. Comput. Sys., 1 (1983), pp. 38-44. 